Assessment of large-eddy simulation in capturing preferential concentration of heavy particles in isotropic turbulent flows

This article has been downloaded from IOPscience. Please scroll down to see the full text article.

2010 Phys. Scr. 2010014061

(http://iopscience.iop.org/1402-4896/2010/T142/014061)

View the table of contents for this issue, or go to the journal homepage for more

Download details:

IP Address: 159.226.230.152

The article was downloaded on 05/01/2011 at 01:58

Please note that terms and conditions apply. 


\title{
Assessment of large-eddy simulation in capturing preferential concentration of heavy particles in isotropic turbulent flows
}

\author{
Guodong Jin ${ }^{1}$, Jian Zhang ${ }^{1}$, Guo-Wei He ${ }^{1}$ and Lian-Ping Wang ${ }^{1,2}$ \\ ${ }^{1}$ LNM, Institute of Mechanics, Chinese Academy of Sciences, Beijing 100190, \\ People's Republic of China \\ ${ }^{2}$ Department of Mechanical Engineering, University of Delaware, Newark, DE 19716-3140, USA \\ E-mail: hgw@lnm.imech.ac.cn
}

Received 26 October 2009

Accepted for publication 2 June 2010

Published 31 December 2010

Online at stacks.iop.org/PhysScr/T142/014061

\begin{abstract}
Particle-laden turbulent flow is a typical non-equilibrium process characterized by particle relaxation time $\tau_{\mathrm{p}}$ and the characteristic timescale of the flows $\tau_{f}$, in which the turbulent mixing of heavy particles is related to different scales of fluid motions. The preferential concentration (PC) of heavy particles could be strongly affected by fluid motion at dissipation-range scales, which presents a major challenge to the large-eddy simulation (LES) approach. The errors in simulated PC by LES are due to both filtering and the subgrid scale (SGS) eddy viscosity model. The former leads to the removal of the SGS motion and the latter usually results in a more spatiotemporally correlated vorticity field. The dependence of these two factors on the flow Reynolds number is assessed using a priori and a posteriori tests, respectively. The results suggest that filtering is the dominant factor for the under-prediction of the PC for Stokes numbers less than 1, while the SGS eddy viscosity model is the dominant factor for the over-prediction of the PC for Stokes numbers between 1 and 10. The effects of the SGS eddy viscosity model on the PC decrease as the Reynolds number and Stokes number increase. LES can well predict the PC for particle Stokes numbers larger than 10. An SGS model for particles with small and intermediate Stokes numbers is needed to account for the effects of the removed SGS turbulent motion on the PC.
\end{abstract}

PACS numbers: 47.27.ep, 47.55.Kf, 47.51.+a

(Some figures in this article are in colour only in the electronic version.)

\section{Introduction}

Turbulent flows laden with heavy particles are found in a wide range of engineering and environmental problems [1]. The density difference between the fluid and an inertial particle induces a relative velocity between the fluid and the particle, which makes the particle-laden turbulent flow a non-equilibrium process characterized by the particle relaxation time $\tau_{\mathrm{p}}$ and the characteristic timescale of the flows $\tau_{f}$. One of the main challenges of modeling such a non-equilibrium process comes from the fact that heavy particles interact with the flow eddies of a wide range of length and timescales [2-6]. The non-equilibrium response of a heavy particle to the turbulent fluid motion depends on the particle Stokes number and gravity. It is now well established that heavy particles tend to accumulate preferentially in regions of high strain rate and low vorticity due to the inertial bias. This phenomenon is termed as the preferential concentration (PC), which has been shown theoretically [7] and validated in experimental and direct numerical simulation (DNS) studies [2, 8]. The PC could greatly enhance the collision rate of heavy particles, which is governed by both small- and large-scale processes in turbulent flows $[3,5,9]$.

In recent years, large-eddy simulation (LES) has emerged as a promising tool for simulating particle-laden 
turbulence [10]. In LES, only the large-scale turbulent motions are explicitly resolved and the effects of the subgrid scale (SGS) turbulent motions on the large-scale ones are modeled using an SGS eddy viscosity model. The SGS turbulent motions are not explicitly simulated. However, the PC could be strongly driven by the small-scale motions, which presents a great challenge to LES of particle-laden turbulent flows $[11,12]$. We have explored the extent to which LES can be used to simulate heavy particle collision and collision-related pair statistics in [5].

The objectives of this paper are to study the effects of flow Reynolds number and to assess separately the effects of the missed SGS turbulent motions and the eddy viscosity model on the PC by comparing the results from the DNS, filtered DNS (FDNS) and LES in isotropic turbulent flows. As a first step, the one-way coupling assumption is used under the condition of dilute particle loadings. The gravitational effect on the PC is not considered. The results show that filtering operation is the dominant factor for the under-prediction of the PC by LES for Stokes numbers of less than 1, and the SGS eddy viscosity model is the dominant factor for the over-prediction of the PC for Stokes numbers of between 1 and 10. Here the Stokes number is defined as the ratio of the particle inertial response time to the flow Kolmogorov timescale, $S t \equiv \tau_{\mathrm{p}} / \tau_{\eta}$. The effects of the SGS eddy viscosity model on the PC decrease as the Reynolds number and Stokes number increase. The LES can well predict the PC for particle Stokes numbers larger than 10 .

The organization of this paper is as follows: the simulation methods for the fluid flow and particle motion are presented in section 2 . The numerical results are discussed in section 3. The conclusions are given in section 4 .

\section{Simulation methods}

The numerical methods for the motions of the fluid and the heavy particles are briefly described in this section.

\subsection{Simulation of turbulent flow}

The forced isotropic turbulent flow fields are simulated using the DNS, FDNS and LES methods, respectively. The turbulent flow is driven and maintained by a random artificial forcing which is non-zero only at low wavenumbers in the Fourier space $[2,13]$. The standard pseudo-spectral method is used to solve the Navier-Stokes equations in the DNS and the filtered Navier-Stokes equations in the LES. The Chollet-Lesieur spectral SGS eddy viscosity model is used for the closure of the filtered Navier-Stokes equations in the LES [14]. Further details of the DNS and LES methods can be found in $[2,5,15]$. The FDNS flow field is obtained from the DNS flow field by filtering out the small-scale fluid motions under a given cut-off wavenumber $k_{\mathrm{cf}}$,

$$
\tilde{\mathbf{u}}(\boldsymbol{x}, t)=\sum_{|\boldsymbol{k}|=k_{0}}^{k_{\mathrm{cf}}} \hat{\boldsymbol{u}}(\boldsymbol{k}, t) \mathrm{e}^{\mathrm{i} \boldsymbol{k} \cdot \boldsymbol{x}},
$$

where $\tilde{\boldsymbol{u}}(\boldsymbol{x}, t)$ is the filtered velocity in the physical space. $k_{0}=1$ is the lowest wavenumber. FDNS can be regarded as an ideal LES to study the effects of SGS eddies on the motions of the heavy particles, since it does not contain any SGS eddy viscosity modeling errors [12].
Table 1. The parameters of the DNS flow fields.

\begin{tabular}{lcc}
\hline Method & DNS $\left(128^{3}\right)$ & DNS $\left(256^{3}\right)$ \\
\hline Molecular viscosity coefficient, $v$ & 0.0062 & 0.0488 \\
rms velocity, $u^{\prime}$ & 0.812 & 19.34 \\
Dissipation rate, $\varepsilon$ & 0.155 & 3739.2 \\
Integral length scale, $L_{\mathrm{f}}$ & 1.65 & 0.99 \\
Kolmogorov timescale, $\tau_{\eta}$ & 0.20 & 0.00361 \\
Kolmogorov velocity scale, $v_{\mathrm{K}}$ & 0.176 & 3.675 \\
Kolmogorov length scale, $\eta$ & 0.0352 & 0.0133 \\
Eddy turnover time, $T_{\mathrm{e}}$ & 2.03 & 0.0519 \\
Length scale ratio, $L_{\mathrm{f}} / \eta$ & 46.83 & 74.436 \\
Timescale ratio, $T_{\mathrm{e}} / \tau_{\eta}$ & 10.15 & 14.366 \\
Velocity ratio, $u^{\prime} / v_{\mathrm{K}}$ & 4.62 & 5.262 \\
Taylor Reynolds number, $R e_{\lambda}$ & 81.6 & 106.05 \\
\hline
\end{tabular}

\subsection{Motion of discrete heavy particles}

The discrete phase is composed of $N_{\mathrm{p}}$ mono-dispersed particles, particle diameter is much less than the turbulent Kolmogorov length scale, $d_{\mathrm{p}} \ll \eta$, and particle density is much larger than that of the carrier fluid, $\rho_{\mathrm{p}} \gg \rho_{\mathrm{f}}$. Neglecting the gravity force, the governing equations for a small heavy particle can be approximated as [2]

$$
\begin{gathered}
\frac{\mathrm{d} \boldsymbol{x}_{\mathrm{p}}(t)}{\mathrm{d} t}=\boldsymbol{v}_{\mathrm{p}}(t), \\
\frac{\mathrm{d} \boldsymbol{v}_{\mathrm{p}}(t)}{\mathrm{d} t}=\frac{\boldsymbol{u}\left(\boldsymbol{x}_{\mathrm{p}}(t), t\right)-\boldsymbol{v}_{\mathrm{p}}(t)}{\tau_{\mathrm{p}}},
\end{gathered}
$$

where $\boldsymbol{x}_{\mathrm{p}}(t)$ and $\boldsymbol{v}_{\mathrm{p}}(t)$ are the particle position and velocity at time $t . \tau_{\mathrm{p}}$ is the particle relaxation time or particle inertial response time. $\boldsymbol{u}\left(\boldsymbol{x}_{\mathrm{p}}(t), t\right)$ is the fluid velocity seen by a heavy particle that is interpolated from the DNS, FDNS and LES flow fields, respectively, using a six-point Lagrangian interpolation scheme in each direction. Equation (3) is integrated using a fourth-order Adams-Bashforth method for particle velocity and equation (2) is then integrated using a fourth-order Adams-Moulton method for particle location.

\section{Results and discussions}

\subsection{Statistics of DNS flow fields}

In order to study the effects of the flow Reynolds number and filter width on the PC, we perform two groups of DNS, FDNS and LES, respectively. We name the first group of DNS $\left(128^{3}\right)$, LES $\left(32^{3}\right)$ and FDNS $\left(k_{\mathrm{cf}}=10\right)$ as case 1 and the second group of DNS $\left(256^{3}\right)$, LES $\left(64^{3}\right)$ and FDNS $\left(k_{\mathrm{cf}}=21\right)$ as case 2 . The cutoff wavenumbers $k_{\mathrm{cf}}=10$ and $k_{\mathrm{cf}}=21$ in FDNS correspond to the space resolutions of $32^{3}$ and $64^{3}$ grids in LES, respectively. Table 1 lists the parameters of the simulated DNS flow fields, where the rms turbulent fluctuation velocity $u^{\prime}$ and the average dissipation rate $\varepsilon$ are computed from the three-dimensional turbulent energy spectrum function, $E(k)$, as

$$
u^{\prime}=\sqrt{\frac{1}{3}\left\langle u_{i} u_{i}\right\rangle}=\sqrt{\frac{2}{3} \int_{k_{0}}^{k_{\max }} E(k) \mathrm{d} k}
$$

and

$$
\varepsilon=\int_{k_{0}}^{k_{\mathrm{c}}} 2 \nu k^{2} E(k) \mathrm{d} k
$$


For isotropic turbulence, the integral length scale $L_{\mathrm{f}}$ is

$$
L_{\mathrm{f}}=\frac{\pi}{2\left(u^{\prime}\right)^{2}} \int_{k_{0}}^{k_{\mathrm{c}}} \frac{E(k)}{k} \mathrm{~d} k .
$$

The Kolomogorov length, time and velocity scales are

$$
\eta=\left(v^{3} / \varepsilon\right)^{0.25}, \quad \tau_{\mathrm{K}}=(\nu / \varepsilon)^{0.5}, \quad v_{\mathrm{K}}=(\varepsilon \nu)^{0.25} .
$$

The Taylor microscale Reynolds number is

$$
R e_{\lambda}=\sqrt{15}\left(u^{\prime} / v_{K}\right)^{2}
$$

The eddy turnover time $T_{\mathrm{e}}$ is defined as

$$
T_{\mathrm{e}}=L_{f} / u^{\prime}
$$

\subsection{Particle PC in DNS, FDNS and LES flow fields}

We compare the PC in the DNS, FDNS and LES flow fields of case 1 to study the effects of small-scale turbulent motions on the local accumulation of heavy particles. Initially, 32000 heavy particles with an identical Stokes number are randomly put into the turbulent flow fields of DNS, FDNS and LES, respectively. The spatial distribution of particles evolves with time, and the PC forms in the flow field when the two-phase flow field reaches a statistically stationary state as shown in figure 1 . The color contours show the vorticity distribution at $y=\pi$ for the DNS $\left(128^{3}\right)$, FDNS $\left(k_{\mathrm{cf}}=10\right)$ and LES $\left(32^{3}\right)$ flow fields, respectively, from left to right. The dots and vectors show the locations and velocities of all particles located within a thin layer $0.96875 \pi<y<1.03125 \pi$, for four Stokes numbers $(S t=0.1,1.0,3.0$ and 10.0). The degree of non-uniformity in the particle distribution depends on the particle Stokes numbers and the flow structures. The spatial resolution of DNS is the finest among the three types of flow fields; therefore there are many small-scale eddies with high vorticity in the DNS field. In the FDNS flow field, the distribution of the large-scale eddies is maintained but the small-scale motions with high vorticity are filtered out. Although the spatial resolution of the LES is the same as that of the FDNS, the flow structures in the LES are more spatiotemporally correlated than those in the FDNS flow field. Furthermore, the vorticity field in the LES is much smoother than that of the FDNS flow field due to the over-dissipation and increased time correlation in LES [16].

When the Stokes number is $S t=0.1$, the particle inertia is small and the particle response time is much shorter than the Kolmogorov timescale; therefore the particles respond quickly to the variation of the flow fields and can nearly follow the streamlines of the flows. However, even with such a small inertial bias, more particles tend to distribute in the regions with low vorticity. As the length scales of the smallest eddies in the FDNS and LES flow fields are larger than those in the DNS flow field, the particle distribution is more homogeneous in the FDNS than that in the DNS and most homogeneous in the LES flow field among the three types of flow fields (see figure 1(a), where the dots represent the particle positions, and the arrows represent the particle velocity vectors). When the particle Stokes number is $S t=1.0$, the particle response time is the same as the Kolmogorov timescale, particle motions are strongly affected by small-scale eddies and are centrifuged out of the eddy kernel and accumulate in the regions of low vorticity. The degree of non-uniformity of particle concentration is maximized at $S t=1.0$ in the DNS flow field. As the velocity in the LES flow field is more coherent in the space than those in the DNS and FDNS flow fields, particles will accumulate in the limited regions of less volume with low vorticity. As a result, the intensity of local accumulation is stronger in the LES flow field than those in the DNS and FDNS flow fields (see figure 1(b)). When the particle Stokes number is $S t=3.0$, the particles respond to eddies with large timescales. As the smallest timescale in the LES flow field is larger than those in the DNS and FDNS flow fields, the particles tend to respond more effectively to eddies in the LES flow field, the particles accumulate more strongly around large-scale eddies in the LES flow field than in the DNS and FDNS flow fields (see figure 1(c)). As the particle Stokes number is further increased, say $S t=10.0$ in figure 1(d), the particles are unable to respond to the evolving flow structures in a timely manner and as a result they tend to distribute uniformly in the flow fields again.

\subsection{Quantification of the PC}

Following [2, 17], a global measure is now used to quantify the PC; it is defined as the summation of the squared deviation between the actual probability of finding a particular particle number of $C(C=0,1,2,3, \ldots)$ in a grid cell, denoted by $P_{\mathrm{c}}(C, t)$, and the corresponding probability for a random distribution, denoted by $P_{\mathrm{c}}^{\mathrm{r}}(C)$, namely

$$
D_{\mathrm{c}}(t)=\sum_{C=0}^{N_{\mathrm{p}}}\left(P_{\mathrm{c}}(C, t)-P_{\mathrm{c}}^{\mathrm{r}}(C)\right)^{2} .
$$

It is obvious that $P_{\mathrm{c}}(C, t)$ depends on the grid size. We use $32^{3}$ grids in case 1 and $64^{3}$ grids in case 2 to compute this probability. At the initial time, the distribution of particles is random, so $P_{\mathrm{c}}(C, t=0)$ satisfies the Poisson distribution, which can be approximated as

$$
P_{C}^{\mathrm{r}}(C)=\frac{\langle C\rangle^{C} \mathrm{e}^{-\langle C\rangle}}{C !} \quad(C=0,1,2,3, \ldots),
$$

where $\langle C\rangle$ denotes the average number of particles per grid cell. As 32000 particles are used in case 1 , so $\langle C\rangle=$ $32000 / 32^{2}=0.9766$, and the probability for each of the first four levels at $t=0.0$ is $P_{\mathrm{c}}^{\mathrm{r}}(C=0)=0.3766, P_{\mathrm{c}}^{\mathrm{r}}(C=$ $1)=0.3678, P_{\mathrm{c}}^{\mathrm{r}}(C=2)=0.1796$ and $P_{\mathrm{c}}^{\mathrm{r}}(C>2)=0.076$. In case 2, 400000 particles are used. Figure 2 shows a typical time evolution of $D_{\mathrm{c}}(t)$ for DNS of case 1 with particle Stokes number $S t=1.0$. At $t=0.0$, the random distribution of particles implies $D_{\mathrm{c}}(t)=0.0$. The increase of $D_{\mathrm{c}}(t)$ with time during the transient period indicates that the particle distribution deviates from the Poisson distribution and the local accumulation of the particles increases with time. Finally, the concentration field approaches a statistically stationary state, where the accumulation and turbulent mixing are balanced. We take the time average of $D_{\mathrm{c}}(t),\left\langle D_{\mathrm{c}}\right\rangle$ to quantify the degree of PC for various Stokes numbers and flow Reynolds numbers in the next subsection. The initial transient period is not used in time averaging. 


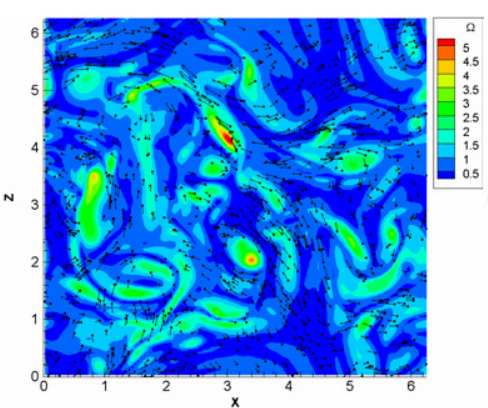

DNS

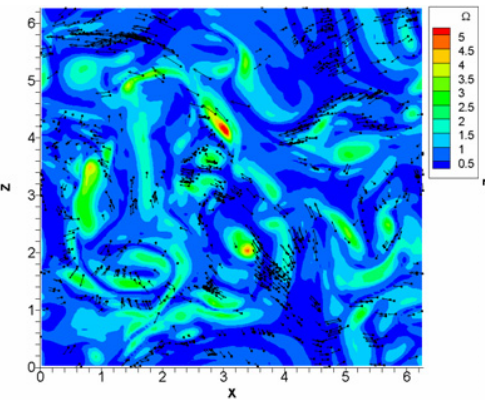

DNS

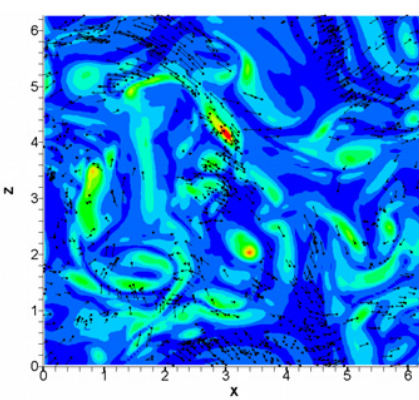

DNS

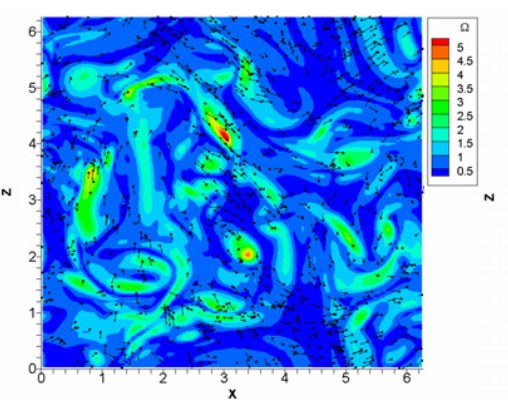

DNS

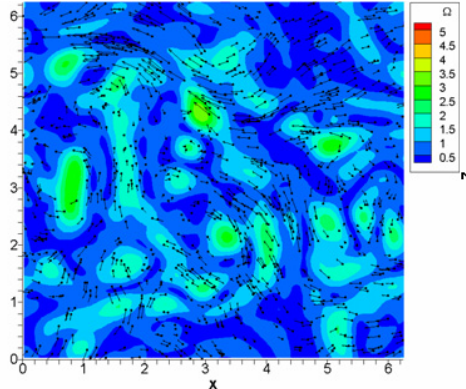

FDNS

(1a) Stokes number $\mathrm{St}=0.1$

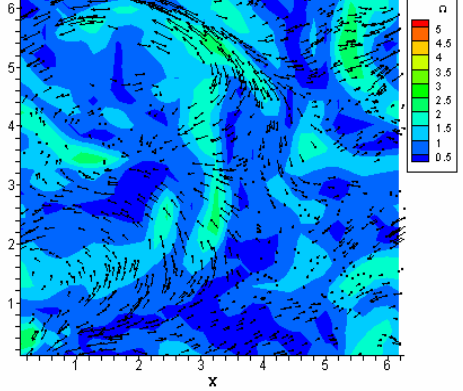

LES

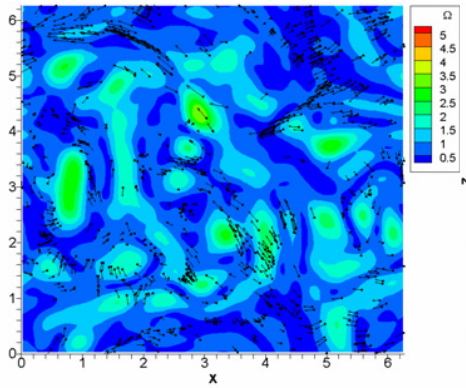

FDNS

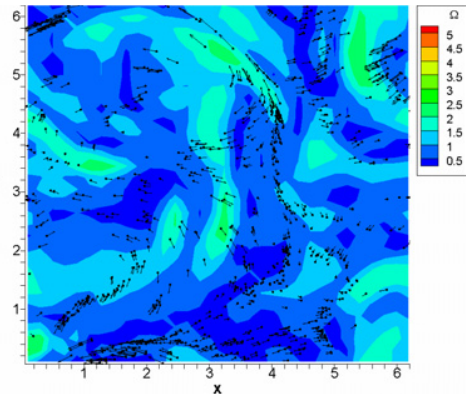

LES

(1b) Stokes number $\mathrm{St}=1$

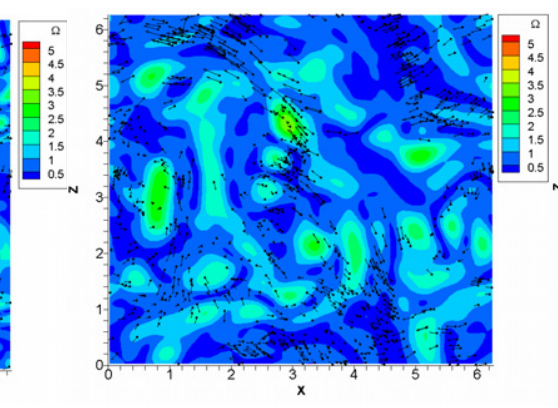

FDNS

(1c) Stokes number $\mathrm{St}=3$

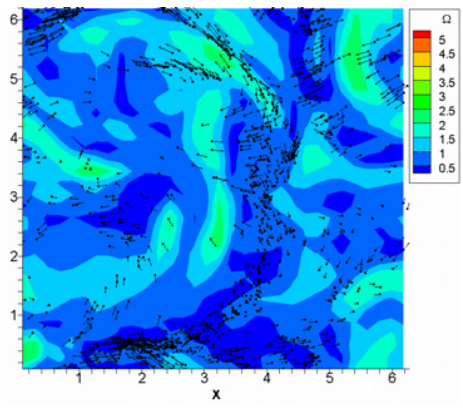

LES

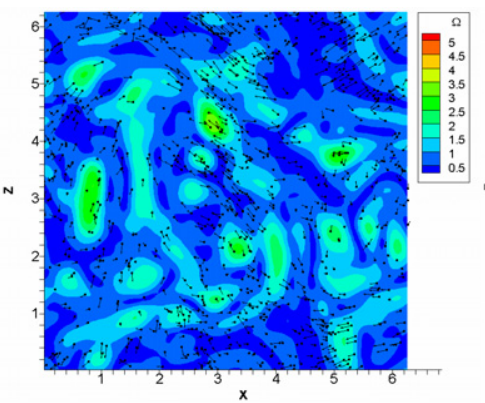

FDNS

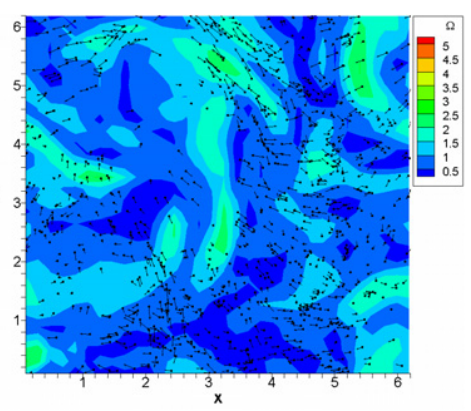

LES

(1d) Stokes number $\mathrm{St}=10$

Figure 1. The PC of heavy particles in the slices of the DNS $\left(128^{3}\right)$, FDNS $\left(k_{\mathrm{cf}}=10\right)$ and LES $\left(32^{3}\right)$ flow fields from the left side to the right, and with different Stokes numbers $S t=0.1,1.0,3.0$ and 10.0 from the top to the bottom. The black dots denote the positions and the arrows denote the velocity vectors of the particles.

\subsection{Comparison of the degree of PC in various flow fields}

As shown in figure 1 , the particle PC is strongly affected by small-scale turbulent eddies which are directly related to the filter width in the FDNS and to both the filter width and the SGS eddy viscosity model in the LES. Figures 3 and 4 show the variations of the time-averaged global measure of PC, $\left\langle D_{\mathrm{c}}\right\rangle$, as a function of particle Stokes number for the DNS, FDNS and LES flow fields at the stationary stage, where the angle brackets denote the time average. The errors 


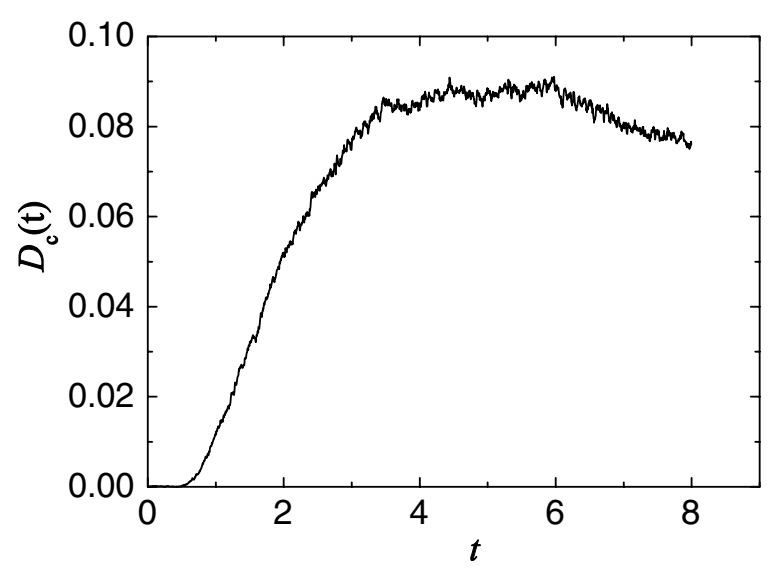

Figure 2. Transient variation of the global measure of local accumulation $D_{\mathrm{c}}(t)$ in the DNS $\left(128^{3}\right)$ flow field and $S t=1.0$.
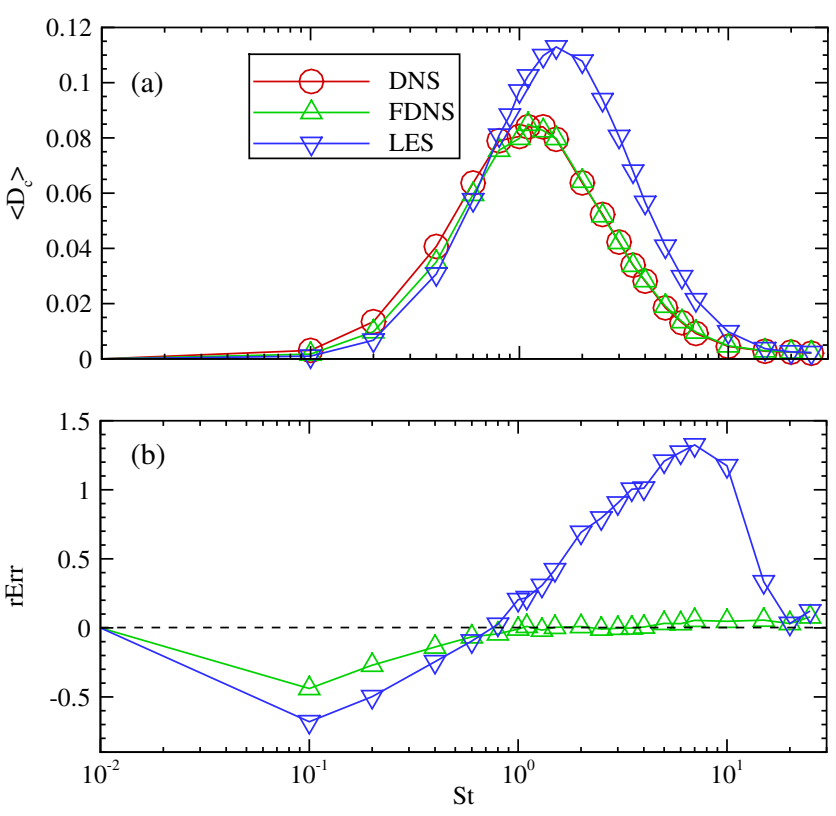

Figure 3. $\left\langle D_{\mathrm{c}}\right\rangle$ and the relative errors of FDNS and LES to DNS versus the Stokes number in case $1\left(R e_{\lambda}=80.0\right)$, where the relative errors are defined as $\left(\left\langle D_{\mathrm{c}}\right\rangle_{\mathrm{LES}}-\left\langle D_{\mathrm{c}}\right\rangle_{\mathrm{DNS}}\right) /\left\langle D_{\mathrm{c}}\right\rangle_{\text {DNS }}$ and $\left(\left\langle D_{\mathrm{c}}\right\rangle_{\mathrm{FDNS}}-\left\langle D_{\mathrm{c}}\right\rangle_{\mathrm{DNS}}\right) /\left\langle D_{\mathrm{c}}\right\rangle_{\mathrm{DNS}}$.

in the FDNS and LES results relative to the DNS results are also shown for a wide range of particle Stokes numbers. From the two figures, we observe that there are three regimes for the behaviors of particle accumulation. For the particle Stokes number $S t \rightarrow 0$ and $S t \rightarrow \infty$, the particles tend to be uniformly distributed and thus $\left\langle D_{\mathrm{c}}\right\rangle \rightarrow 0$. These limiting behaviors are expected, because the particles with small Stokes numbers are distributed like fluid elements, while the particles with very large Stokes numbers have a chaotic motion with little response to evolving fluid flow eddies. For intermediate Stokes numbers, the particles interact with a range of fluid eddies and tend to accumulate in the regions of low vorticity, leading to larger values of $\left\langle D_{\mathrm{c}}\right\rangle$. Figures 3 and 4 show that both FDNS and LES under-predict $\left\langle D_{\mathrm{c}}\right\rangle$ when particle Stokes numbers are less than 1.0, and the dominant factor contributing to the relative error comes from the filtering effect. The reason is that the PC of the particles with small Stokes numbers are mainly driven by small-scale eddies. Filtering operation in the FDNS and LES flow fields
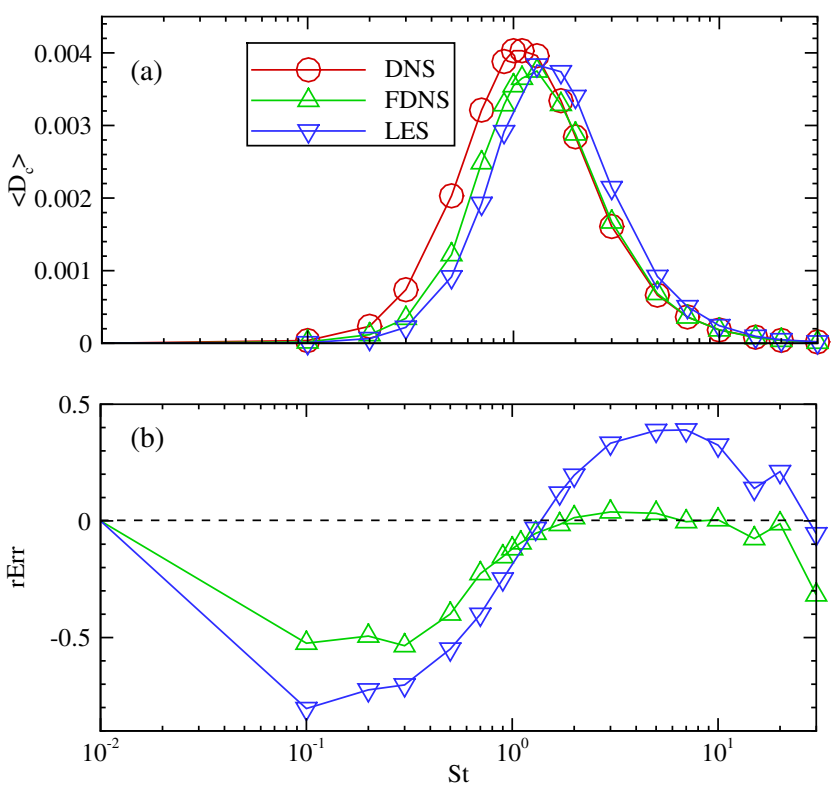

Figure 4. $\left\langle D_{\mathrm{c}}\right\rangle$ and the relative errors of FDNS and LES to DNS versus the Stokes number in case $2\left(R e_{\lambda}=102.1\right)$, where the relative errors are defined as $\left(\left\langle D_{\mathrm{c}}\right\rangle_{\mathrm{LES}}-\left\langle D_{\mathrm{c}}\right\rangle_{\mathrm{DNS}}\right) /\left\langle D_{\mathrm{c}}\right\rangle_{\mathrm{DNS}}$ and $\left(\left\langle D_{\mathrm{c}}\right\rangle_{\mathrm{FDNS}}-\left\langle D_{\mathrm{c}}\right\rangle_{\mathrm{DNS}}\right) /\left\langle D_{\mathrm{c}}\right\rangle_{\mathrm{DNS}}$.

weakens the intensity of small-scale eddies and thus reduces the PC. However, the FDNS and LES over-predict $\left\langle D_{\mathrm{c}}\right\rangle$ when the particle Stokes numbers are between 1.0 and 10.0. The relative error in the LES results is much larger than that of the FDNS. The dominant factor contributing to the relative error comes from the SGS eddy viscosity model. The PC of the particles with intermediate Stokes numbers is driven by a wide range of length scales of eddies including small-scale dissipative eddies and large-scale energy-containing ones. Small-scale eddies act as random forces to mix the particles and to reduce the PC, while large-scale eddies enhance the $\mathrm{PC}$ of such particles. Thus, filtering out random small-scale eddies in the FDNS and LES increases the PC. As the SGS eddy viscosity model is developed under the assumption of high flow Reynolds number, it is usually over-dissipative for turbulent flows with moderate flow Reynolds numbers. The over-dissipation makes the vorticity field obtained from the LES much smoother than that in the FDNS with similar space resolutions. Therefore, the relative error of $\left\langle D_{c}\right\rangle$ in the LES flow field is much larger than that in the FDNS flow field. One can further observe that the relative error of $\left\langle D_{\mathrm{c}}\right\rangle$ in the LES decreases as the turbulent Reynolds number increases. For example, the maximum relative error of $135 \%$ in figure 3 reduces to $39 \%$ in figure 4 . For particles with Stokes numbers larger than 10.0, small-scale eddies have little effect on the motions of particles; thus, LES can well predict the PC of particles at large Stokes numbers.

Our results about the effects of filtering operation and particle inertia on the PC are consistent with the work of Fede and Simonin [12] for the three Stokes number ranges, which represent the different contributions of small-scale and large-scale turbulent motions and particle inertia to the PC. Here, in addition, we have addressed separately the effects of SGS eddy viscosity modeling and flow Reynolds number. 


\section{Conclusion}

In the non-equilibrium particle-laden turbulent flows, heavy particles interact with the flow eddies of a wide range of lengths and timescales. The removal of the SGS turbulent motions due to the filtering and the SGS eddy viscosity model error are the two sources contributing to the errors in the LES of the PC. These two factors are separately assessed using the FDNS and LES flows at the same spatial resolution. The degree of PC predicted by LES shows three different regimes: the under-prediction of $\left\langle D_{\mathrm{c}}\right\rangle$ for $S t<1$ primarily due to the filtering effect, the over-prediction of $\left\langle D_{\mathrm{c}}\right\rangle$ for $1<S t<10$ primarily due to the SGS eddy viscosity model error and an acceptable prediction of $\left\langle D_{\mathrm{c}}\right\rangle$ for $S t>10$. Fortunately, we demonstrate that the effects of the SGS eddy viscosity model error on the relative error in the predicted $\left\langle D_{\mathrm{c}}\right\rangle$ decreases with increasing flow Reynolds number and particle Stokes number. The assessment of the effects of the two factors and flow Reynolds number in LES helps us to clarify the main factors influencing the prediction of the PC for particles at different Stokes numbers. New SGS models including particle-flow interactions are needed to account for the filtering effect and SGS model error in order for LES to be a reliable approach for particle-laden turbulence.

\section{Acknowledgments}

This work was supported by CAS (KJCX2-SW-L08), 973 Program of China (2007CB814800 and 2009CB724100), NSFC (110772247, 10732090, 10702074 and 50906096), the
LNM initial funding for young investigators and the Scientific Research Foundation for the Returned Overseas Chinese Scholars, State Education Ministry. LPW acknowledges support from the US National Science Foundation (grants ATM-0527140 and ATM-0730766) and computing resources support from National Center for Atmospheric Research (CISL-35751010 and CISL-35751014).

\section{References}

[1] Crowe C T 2006 Multiphase Flow Handbook (Boca Raton, FL: Taylor and Francis)

[2] Wang L-P and Maxey M R 1993 J. Fluid Mech. 25627

[3] Wang L-P, Wexler A S and Zhou Y 2000 J. Fluid Mech. 415117

[4] Jin G D, He G-W, Wang L-P and Zhang J 2010 Int. J. Multiph. Flow 36432

[5] Jin G D, He G-W and Wang L-P 2010 Phys. Fluids 22055106

[6] Yoshimoto H and Goto S 2007 J. Fluid Mech. 577275

[7] Maxey M R 1987 J. Fluid Mech. 174441

[8] Wood A M, Hwang W and Eaton J K 2005 Int. J. Multiph. Flow 311220

[9] Sundaram S and Collins L 1997 J. Fluid Mech. 33575

[10] Wang Q and Squires K D 1996 Phys. Fluids 81207

[11] Armenio V, Piomelli U and Fiorotto V 1999 Phys. Fluids 113030

[12] Fede P and Simonin O 2006 Phys. Fluids 18045103

[13] Eswaran E and Pope S B 1988 Comput. Fluids 16257

[14] Chollet J-P and Lesieur M 1981 J. Atmos. Sci. 382747

[15] Yang Y, He G-W and Wang L-P 2008 J. Turbulence 98

[16] He G-W, Rubinstein R and Wang L-P 2002 Phys. Fluids 142186

[17] Squires K-D and Eaton J K 1991 Phys. Fluids A 31169 